\title{
Calibration Transfer for Food Recognition Models for E-Noses
}

\author{
Andreas Seiderer \\ Augsburg University \\ Augsburg, Germany \\ seiderer@hcm-lab.de
}

\author{
Chi Tai Dang, Elisabeth André \\ Augsburg University \\ Augsburg, Germany \\ \{dang,andre\}@informatik.uni-augsburg.de
}

\section{ABSTRACT}

Gas sensors using the low-priced MOS technique face several problems that reduce their applicability for classification tasks. In literature, several methods can be found that try to alleviate these problems In this extended abstract, we focus on the problem that the behavior of different sensors (of same type) results in different data even for the same gas concentrations. Hence, the models cannot be reused for sensors of same type. Additionally, it is hardly possible to record high amounts of this type of data which makes transfer learning techniques on such data sets necessary. We apply a calibration transfer procedure and present the results on a data set for food recognition, which we recorded simultaneously with two identical gas sensor boxes in a controlled environment. We compare the results on MOS-based gas sensors from the MQ series and a more modern sensor using MEMS fabrication. The applied technique shows increased recognition rates on this data set.

\section{CCS CONCEPTS}

- Computing methodologies $\rightarrow$ Transfer learning; Support vector machines; • Hardware $\rightarrow$ Sensor applications and deployments.

This is the author's own version. It is posted here for your personal use. Not for redistribution.

For all other uses, contact the owner/author(s).

IoT 2019, October 22-25, 2019, Bilbao, Spain

(c) 2019 Copyright held by the owner/author(s).

ACM ISBN 978-1-4503-7207-7/19/10.

https://doi.org/10.1145/3365871.3365910 


\begin{tabular}{|l|l|}
\hline Foodstuff & Type \\
\hline apple & fruit \\
\hline banana & fruit \\
\hline black tea & hot drink \\
\hline buttermilk & dairy product \\
\hline carrot juice & vegetable juice \\
\hline chardonnay & white wine \\
\hline coffee & hot drink \\
\hline cola & soft drink with $\mathrm{CO}_{2}$ \\
\hline dornfelder & red wine \\
\hline emmenthaler & cheese \\
\hline garlic & vegetable \\
\hline grapefruit juice & fruit juice \\
\hline green tea & hot drink \\
\hline herder's cheese & cheese \\
\hline hot tap water (no chlorine) & water \\
\hline merlot & red wine \\
\hline milk & dairy product \\
\hline onion & vegetable \\
\hline orange juice & fruit juice \\
\hline pear & fruit \\
\hline quark & dairy product \\
\hline sparkling wine / champagne & white wine with $\mathrm{CO}_{2}$ \\
\hline tap water (no chlorine) & water \\
\hline tomato juice & vegetable juice \\
\hline
\end{tabular}

Table 1: The 24 recorded foodstuff classes.

\section{KEYWORDS}

e-nose, gas sensors, MOS, MEMS, food recognition

\section{ACM Reference Format:}

Andreas Seiderer and Chi Tai Dang, Elisabeth André. 2019. Calibration Transfer for Food Recognition Models for E-Noses. In 9th International Conference on the Internet of Things (IoT 2019), October 22-25, 2019, Bilbao, Spain. ACM, New York, NY, USA, 4 pages. https://doi.org/10.1145/3365871.3365910

\section{INTRODUCTION}

Gas sensors using the low-priced MOS technique face several issues that reduce their applicability for classification tasks, e.g. sensor drift, dependency to other gases, and temperature / humidity. In addition, they usually show unique behaviors making them hard to use models of one sensor even with a gas sensor of the same type. In this work, we evaluate a recent calibration transfer technique by Laref et al. [3] for the last problem. We applied this technique to a food stuff classification task and present the results of our experiments.

\section{HARDWARE AND DATA SET}

For the calibration transfer experiment we used the data set which was introduced by Dang et al. [2]. A picture of the recording setup with the two sensor boxes (e-noses) and a sample in the open container is depicted in Figure 1. For the experiments we focused on the MQ series (MQ2, MQ3, MQ5, MQ9) and the MEMS-fabricated (microelectromechanical systems) MiCS-6814 sensors which were provided by each sensor box. All data were recorded by both e-noses in parallel with a synchronized system clock. Data was acquired for each of the 24 food stuff samples over about three minutes in a closed container. The measurements were repeated two times after each other. After each measurement the container was sufficiently aired. The recorded food stuff samples are listed in Table 1.

All data were up-sampled with their timestamps to a one-second resolution for synchronization. This resulted in about 400 data values per sensor box for each sensor and sample class.

\section{CALIBRATION TRANSFER PROCEDURE}

For the calibration transfer we used an adapted technique to the one presented in the work by Laref et al. [3]. Since we did not focus on the reduction of required data, we did not use the kernel SPXY for the selection of standardized samples. Additionally, we used the data for a classification task instead of calculating the absolute gas concentrations. Thus, we used a (SVM) classificator in the last step.

Figure 2 shows our calibration transfer process. The resulting confusion matrices are given in Figure 3 where Figure 2 a) is shown in the left column, b) in the middle and c) on the right. 


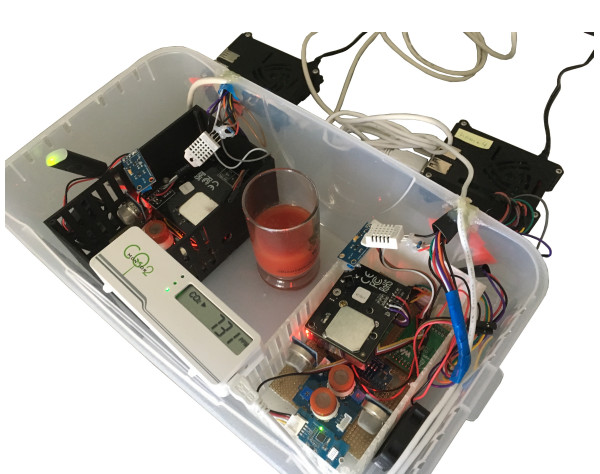

Figure 1: Measurement setup.

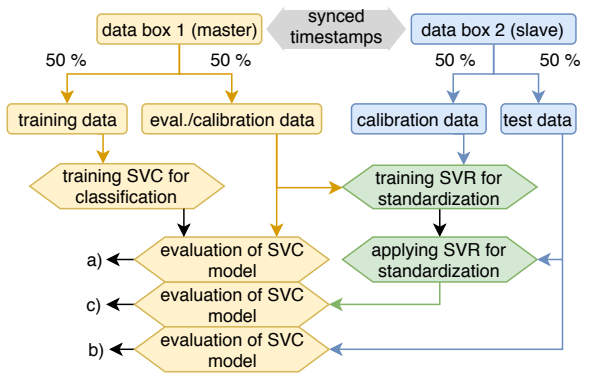

Figure 2: Calibration transfer process. The results of a) to c) can be found in Figure 3.
In this case, sensor box 1 is considered as "master" whose model should be used on the data of sensor box 2 ("slave"). At first, the synchronized data of each box is split into two parts of equal amounts of data. The training data of the master was applied to train the SVM classification model (SVC). The eval./calibration data was used to evaluate the classification model and for the training of the SVM regression model (SVR). For the training of the regression model the synchronized calibration data of the slave was also required. The other half of the data of the slave was utilized as test data. The trained regression model was applied on this data for standardization. For a comparison the non-standardized and standardized test data were used with the SVM classification model.

\section{EVALUATION}

In the following, the SVM c and gamma parameters were tested in each combination for the ranges: $\left[10^{-7} ; 10^{7}\right]$ with a step of 10 (225 combinations). For the MiCS sensor, the combinations resulting in the highest precision were chosen as they showed also a high recall. For the MQ2 sensor, the parameters were optimized for precision and recall (maximized sum).

Of the MiCS-6814 sensor just the raw values of the three sensor components (RED, OX, NH3 [1]) were used. The confusion matrices are visible at the top of Figure 3. For the MQ sensors, the data of the MQ2 sensor achieves the model with the highest precision and recall after calibration transfer for our data set. A combination resulted in lower performance after the transfer so that we just provide the results of the MQ2 sensor at the bottom of Figure 3.

\section{CONCLUSION}

Our results show that the calibration transfer technique allows a high improvement of model performance also for a classification task. Especially, the MiCS sensor took profit from this approach which can be integrated into compact / mobile devices. Nevertheless, there is still room for improvement and other techniques should be tested in future that don't require high data amounts.

\section{ACKNOWLEDGMENTS}

The authors would like to thank the student Alex Barth who contributed to this work.

\section{REFERENCES}

[1] [n.d.]. Data Sheet SGX SENSORTECH MiCS-6814. https://www.sgxsensortech.com/content/uploads/2015/02/1143_ Datasheet-MiCS-6814-rev-8.pdf Accessed July 2, 2019.

[2] Chi Tai Dang, Andreas Seiderer, and Elisabeth André. 2018. Theodor: A Step Towards Smart Home Applications with Electronic Noses. In Proceedings of the 5th International Workshop on Sensor-based Activity Recognition and Interaction (iWOAR '18). ACM, New York, NY, USA, Article 11, 7 pages. https://doi.org/10.1145/3266157.3266215

[3] Rachid Laref, Etienne Losson, Alexandre Sava, and Maryam Siadat. 2018. Support Vector Machine Regression for Calibration Transfer between Electronic Noses Dedicated to Air Pollution Monitoring. Sensors 18, 11 (2018). 

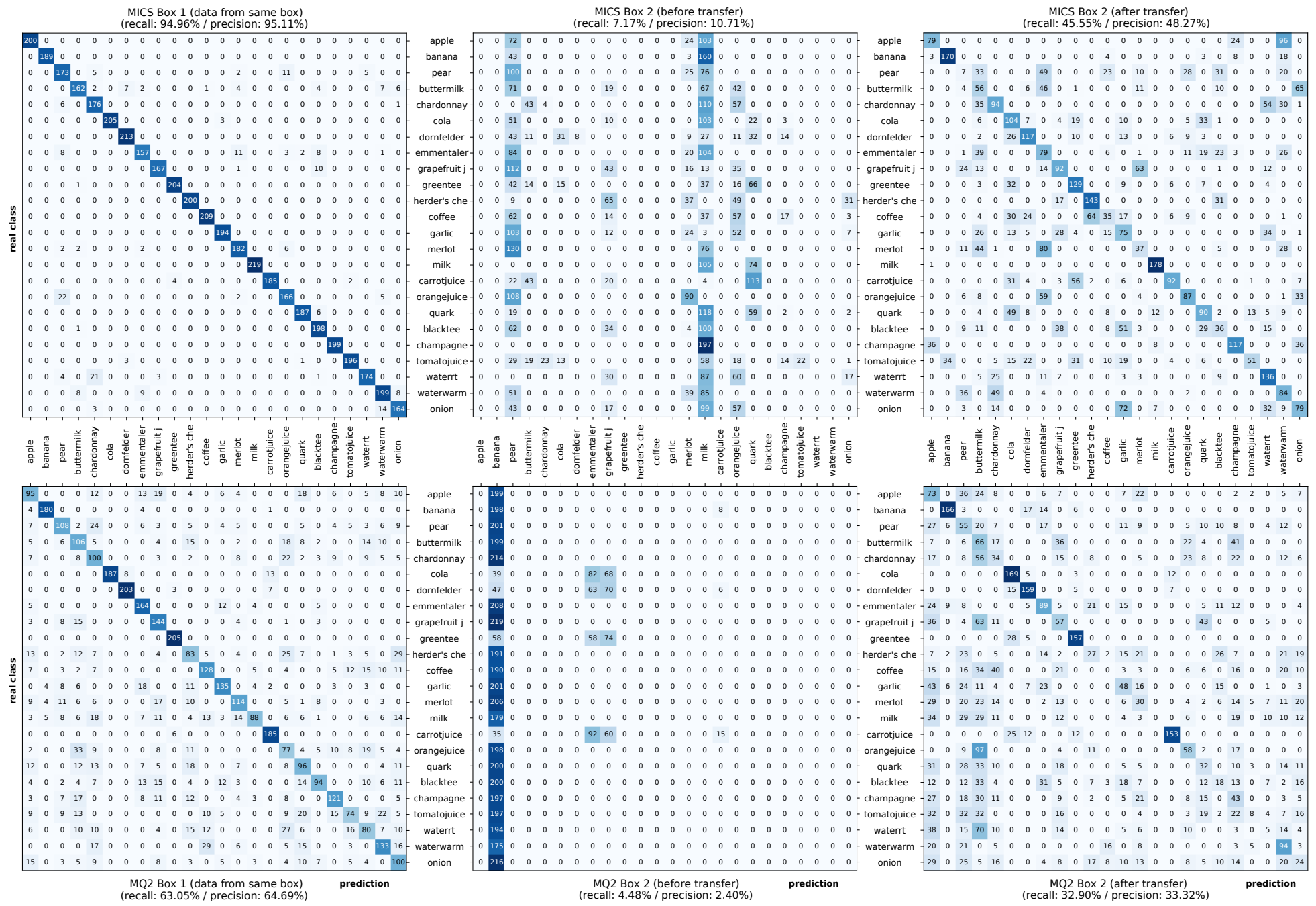

Figure 3: Classification results with evaluation data of the same sensor box (box 1$)$, before and after calibration transfer. $($ waterrt $=$ water room temperature). 